\title{
Fusion reactions induced by halo and weakly bound nuclei around the Coulomb barrier: results and experimental problems
}

\section{P. Figuera ${ }^{1}$}

INFN - Laboratori Nazionali del Sud

Via S. Sofia 62, I-95123 Catania, Italy

E-mail: figueraelns.infn.it

The study of collisions around the Coulomb barrier, induced by halo and weakly bound nuclei, has been the object of many publications in the last decade. In fact, it is expected that the low breakup threshold, coupled to the halo or cluster structure of the ground state of the projectiles, can deeply affect the reaction dynamics. In this paper, we will give an overview of our present understanding in the study of fusion reactions induced by such beams, by comparing some of our experimental results with ones of other authors. The need of new better quality data for a deeper understanding of the discussed topic will be underlined, and some of the related experimental challenges discussed.

X Latin American Symposium on Nuclear Physics and Applications (X LASNPA)

December 1-6 2013

Montevideo, Uruguay

1

Speaker 


\section{Introduction}

In the last decades, with the availability of radioactive ion beams (RIBs), a lot of experiments have been devoted to the study of collisions induced by halo and/or weakly bound nuclei. In fact, the very low breakup threshold of such nuclei combined with the halo or cluster structure of the ground state, can strongly affect the reaction dynamics around the Coulomb barrier. [e.g. 1-6]. For such nuclei, direct reaction processes such as breakup or transfer can be favored by the low breakup threshold coupled with the cluster or halo structure. In addition, since the continuum of such nuclei is very close to the ground state, coupling to continuum effects become important and a complete theoretical description of such collisions requires Continuum Discretized Coupled Channels (CDCC) calculations.

Fusion excitation functions induced by such nuclei may be affected by static and dynamic effects. Static effects are due to the fact that the diffuse surface of these nuclei affects the shape of the projectile-target potential reducing the average Coulomb barrier, therefore potentially enhancing the fusion cross section. On the other hand, coupled channel effects dominating fusion below the barrier, present here a new aspect due to the effects of coupling to continuum. In addition, such fusion reactions are complicated by the fact that, due to the large breakup probability of the projectiles, together with complete fusion $(\mathrm{CF})$ one can have a non negligible contribution of incomplete fusion (ICF) following breakup of the projectile. In order to understand how the above mentioned problems affect the fusion excitation functions around the barrier, a lot of experiments have been performed.

In the following, combining some of our experimental results with ones of other authors, we will give an overview of our present understanding of the discussed topic and related experimental challenges.

\section{Measuring fusion cross sections in collisions induced by halo or weakly bound nuclei}

Fusion cross sections are usually measured either directly detecting the fission fragments (FF) or evaporation residues (ER) produced in the compound nucleus (CN) de-excitation, or detecting the gamma rays emitted in the de-excitation cascade. However, for collisions induced by the beams considered in the present paper, a different experimental technique has mainly been used. For reactions on very heavy targets the conventional detection of the produced FF has been used, and high efficiency detection systems compensated the low currents of the radioactive beams [e.g. 7,8]. However, fusion reactions induced by a beam of light nuclei, such as the halo and stable weakly bound nuclei considered in present paper, on a medium mass target at energies around and below the barrier, will lead to production of low-energy evaporation residues (ER). Due to their low energy, direct detection and identification of the produced ER can be extremely difficult and, in some cases, a large fraction of them may even not come out from the target, since their energies are too small. For these reasons, fusion cross sections for such systems have been measured mainly by using activation techniques [e.g. 9-14], which measure the E.R. yield via the off-line detection of $\gamma$-rays, X-rays and $\alpha$ particles produced in their radioactive decay. In our group, we concentrated on reactions producing ER decaying by electron capture (EC) detecting off line the atomic X-rays following the EC decay of the ER. As an example, in figure 1(a) we show an off line X-ray spectrum measured for the collision ${ }^{6} \mathrm{Li}+{ }^{64} \mathrm{Zn}$ at $\mathrm{E}_{\text {lab }} 20 \mathrm{MeV}$. Peaks corresponding to the decay of different ER identified in charge are clearly visible. Since different isotopes of the same element have different half lives, following the activity of each peak as function of time one can unfold the different 
contributions, as shown in figure 1(b) for the Ga isotopes. Finally, analyzing the activity curves, the production cross sections for the different ER, and thus the fusion cross section, can be extracted. Details of the used procedure can be found in [14].
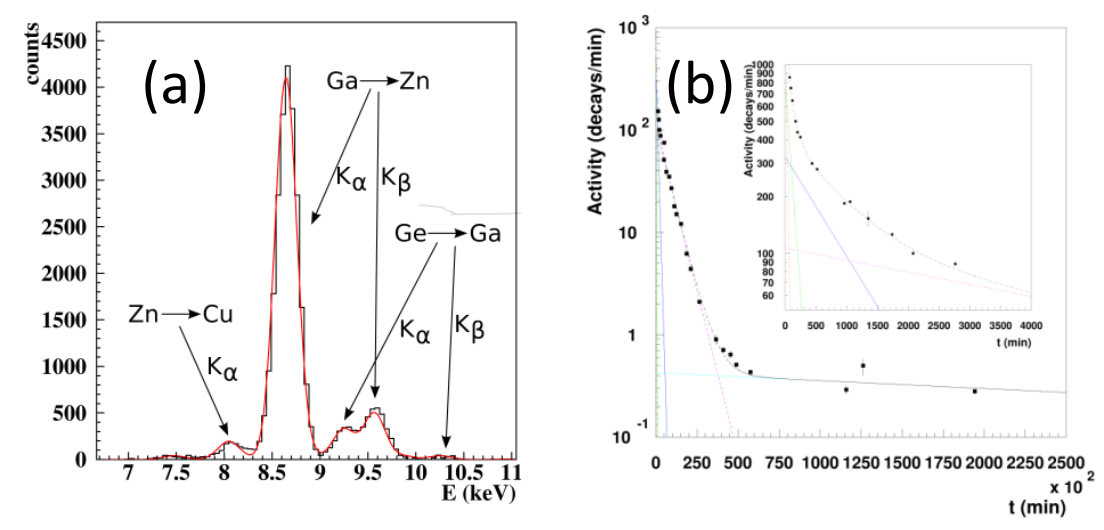

Figure 1. (a) Off line X-ray energy spectrum measured in the collision ${ }^{6} \mathrm{Li}+{ }^{64} \mathrm{Zn}$ at $\mathrm{E}_{\mathrm{LAB}}=20 \mathrm{MeV}$, about 7 hours after the end of the activation. $\mathrm{K} \alpha$ and $\mathrm{K} \beta$ lines corresponding to the decay of $\mathrm{Zn}, \mathrm{Ga}$ and Ge isotopes are clearly visible. (b) Activity curve for the $\mathrm{Ga} \mathrm{K} \alpha$ peak for the collision ${ }^{7} \mathrm{Li}+{ }^{64} \mathrm{Zn}$ at $\mathrm{E}_{\text {c.m. }}=28.3 \mathrm{MeV}$. Different slopes corresponding to known half-lives of the produced Ga isotopes are clearly visible.

A widely used solution, suitable when using activation techniques for this kind of beams, is to irradiate a group of targets each one alternated with a catcher/degrader foil [e.g. 9-13]. Such foil is used to stop the small fraction of residues which emerge from the previous target and to increase the difference in beam energy for the different targets, thus allowing to measure more points in a fusion excitation function using a fixed beam energy. This allows to greatly reduce the beam time needed to perform an excitation function measurement with very low intensity radioactive beams. The disadvantage of using this technique is that the beam energy distribution will degrade as the beam traverses the different stack elements. Therefore, since fusion excitation functions below the barrier have a strong energy dependence, in order to obtain reliable results, the beam energy distribution impinging on each target of the stack has to be properly evaluated, taking into account both the statistical energy straggling and the foil non uniformity effects.

\section{Fusion reactions in collisions induced by halo nuclei}

Different results have been presented in the literature concerning fusion reaction studies, around the Coulomb barrier, in collision induced by halo nuclei [e.g. 7-13]. As an example, in our group we measured, in two independent experiments performed at the CRC-Louvain La Neuve, fusion excitation functions around the Coulomb barrier for collisions induced by the n-halo nucleus ${ }^{6} \mathrm{He}$ and its core ${ }^{4} \mathrm{He}$ on a ${ }^{64} \mathrm{Zn}$ target [9]. The excitation functions have been measured irradiating two stacks of four targets each and by detecting off line at the atomic XRays following the EC decay of the residues, as explained in previous section. In Figure 2(a) we compare the ${ }^{6} \mathrm{He}+{ }^{64} \mathrm{Zn}$ and ${ }^{4} \mathrm{He}+{ }^{64} \mathrm{Zn}$ experimental fusion excitation functions. As one can see, an enhancement of fusion for the ${ }^{6} \mathrm{He}$ induced collision, with respect to the one induced by ${ }^{4} \mathrm{He}$, is observed in the region around the barrier $\left(\mathrm{V}_{\mathrm{B}} \approx 9 \mathrm{MeV}\right)$. Static effects on the fusion excitation function, due to the extended matter distribution in ${ }^{6} \mathrm{He}$, can be removed by 
comparing reduced fusion excitation functions i.e. $\left(\sigma / \pi R_{B}^{2}\right)$ versus $\left(E_{c m}-V_{B}\right)$ were $V_{B}$ and $R_{B}$ are the barrier and barrier radius extracted from potentials calculated with realistic densities, as suggested in $[15,16]$. By performing such comparison, as shown in figure $2 \mathrm{~b}$, the observed enhancement disappears. Therefore, one can conclude that it can be explained as mainly due to static effects.
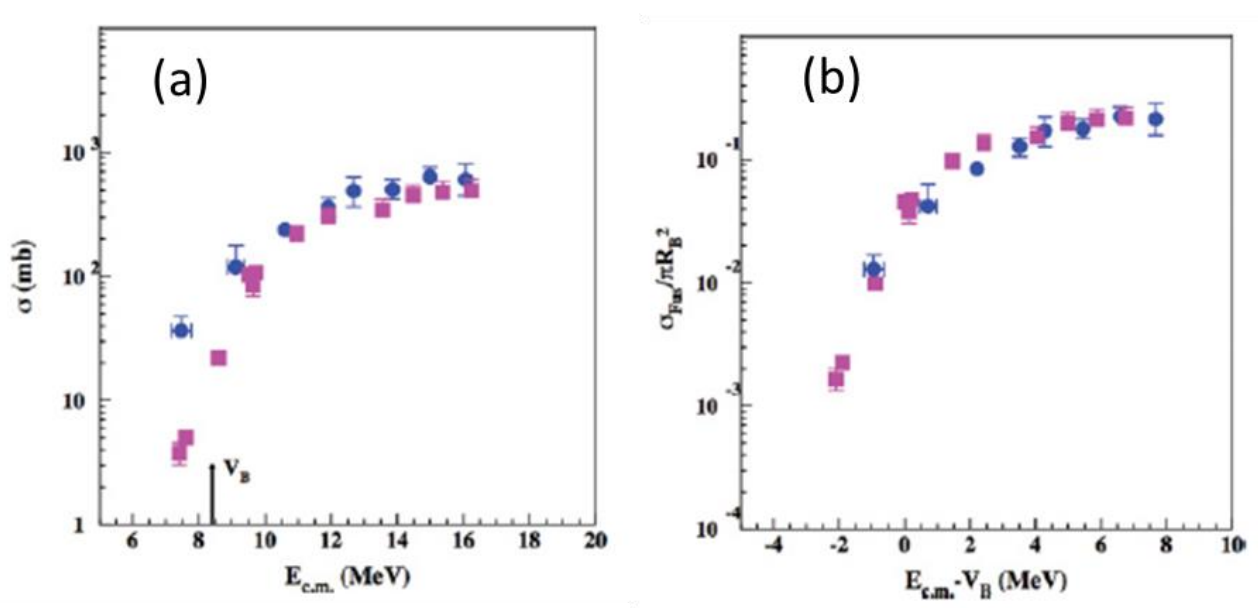

Figure 2. (a) Fusion excitation functions for ${ }^{6} \mathrm{He}+{ }^{64} \mathrm{Zn}$ (blue circles) and ${ }^{4} \mathrm{He}+{ }^{64} \mathrm{Zn}$ (purple squares). The arrow indicates the position of the Coulomb barrier. (b) Reduced fusion excitation functions eliminating static effects for ${ }^{6} \mathrm{He}+{ }^{64} \mathrm{Zn}$ (blue circles) and ${ }^{4} \mathrm{He}+{ }^{64} \mathrm{Zn}$ (purple squares). See text for details.

Enhancement of fusion cross sections around the barrier in collisions induced by n-halo nuclei, with respect to collisions induced by the halo nucleus core, or with respect to calculations based on potentials which do not take into account the diffuse structure of the projectiles, has now been found for different systems in the literature [e.g. 8-13]. In addition, the relative yield of the ER, for those data sets were it was measured, is in good agreement with the predictions of statistical model calculations for complete fusion. Therefore, to our opinion, complete fusion appears to be the dominating mechanism whereas the incomplete fusion of the core gives a marginal contribution.

In the last years, a coherent systematic comparison of many published fusion data, in collisions induced by halo and weakly bound nuclei on targets of different mass, has been presented in the literature $[15,16]$. Such a systematic study suggests that, for collisions induced by neutron halo nuclei, once static effects are removed, the residual effect of coupling to continuum or transfer is a suppression of fusion above barrier and, most probably, a residual small enhancement of fusion below the barrier.

Even if systematic effects of the n-halo on fusion starts to be clear, most of presently available data were obtained with ${ }^{6} \mathrm{He}$ beams and do not explore the sub-barrier region with reasonable errors. Therefore, new fusion data better exploring the low energy region, possibly also with nhalo beams different from ${ }^{6} \mathrm{He}$, would be necessary for a more complete understanding of this topic. This, however, is very difficult due to the low intensities of radioactive ion beams presently available.

Recently, new data concerning the fusion of the proton halo nucleus ${ }^{8} \mathrm{~B}$ on ${ }^{58} \mathrm{Ni} \quad[17]$ and ${ }^{28} \mathrm{Si}$ [18] have also been presented and discussed [19] in the literature. Unfortunately, the conclusions of these studies on the proton halo effect on fusion are not in agreement and, once more, new experimental data are necessary for a better understanding. 


\section{Fusion reactions in collisions induced by weakly bound nuclei with no halo}

Fusion excitation functions in collisions induced by weakly bound nuclei with no halo structure, such as the stable ${ }^{6} \mathrm{Li},{ }^{7} \mathrm{Li},{ }^{9} \mathrm{Be}$, have been measured for several systems in a wide target mass range [e.g. 14, 20-27]. In fusion-evaporation reactions induced by weakly bound nuclei on heavy targets [e.g. 20-22], the emission of charged particles is suppressed by the presence of a strong Coulomb barrier. Therefore, the charge $\mathrm{Z}$ of the produced ERs is the same of the compound nucleus. As a consequence, CF and ICF contributions can be usually clearly separated provided the produced ER are charge identified. The main result, observed for such systems by the different authors, is that CF is suppressed above the barrier by about $30 \%$ with respect to the prediction of Single Barrier Penetration (SBP) calculations or Coupled Channel (CC) calculations not taking into account coupling to continuum. Since the sum of CF and ICF, usually called total fusion (TF), is not suppressed, it was suggested in the literature that the observed suppression is mainly due to breakup of the projectiles followed by ICF. The fusion systematic study of $[15,16]$ mentioned in section 3, confirms this CF fusion suppression above barrier and also suggest a possible CF enhancement below the barrier.

In the fusion of weakly bound nuclei on medium mass or light targets [e.g. 23-27], due to the lower Coulomb barrier, the compound nucleus can evaporate charged particles. Therefore, the $\mathrm{CF}$ and ICF mechanisms can populate the same ERs, making their separation much more difficult. For this reason, most of the fusion data for light and medium mass systems are relative to TF cross section which, as for collisions on heavy targets, do not show any suppression or enhancement effect above barrier, whereas below the barrier not much data are available. Recently, we studied the collisions ${ }^{6,7} \mathrm{Li}+{ }^{64} \mathrm{Zn}$ around the Coulomb barrier, measuring the excitation functions for heavy residue (HR) production. The experiment has been performed at INFN-LNS Catania with the same activation technique mentioned in section 2, the experimental details are reported in [14]. The excitation functions for HR production were compared with SBP calculations and CC calculations including the excitation of different states of projectile and target. Both calculations reproduce the data above barrier but not below it. The experimental relative yield of the HR was compared with different statistical model calculations, performed using the code Cascade, in order to investigate on the reaction mechanisms responsible for their production. ${ }^{6} \mathrm{Li}$ and ${ }^{7} \mathrm{Li}$ are both weakly bound with $\alpha-\mathrm{d}$ and $\alpha-t$ cluster structures of the ground state and corresponding separation energies $S_{\alpha}=1.47 \mathrm{MeV}$ and $\mathrm{S}_{\alpha}=2.47 \mathrm{MeV}$ respectively. Therefore, one can expect to observe reactions where only an alpha particle, a deuteron or a tritium are captured by the target. This may happen in two different ways, breakup of the projectile followed by incomplete fusion (ICF) or a direct cluster transfer (DCT). Since ICF and DCT populate the same nuclei with similar excitation energies, we cannot distinguish between the two modes in this experiment. Expected relative yields of the HR populated in ICF or DCT have been calculated with the statistical model code Cascade. The excitation energies following the $\mathrm{d}, \mathrm{t}$ or $\alpha$ capture were estimated assuming an ICF mechanism where the available fragment-target centre of mass energy before ICF occurs is: $\left(E_{c . m .}-S_{\alpha}\right) \times\left(m_{\text {clu }} / m_{\text {proj }}\right)$. Here $E_{c . m .}$ is the initial centre of mass energy of the $\mathrm{Li}+{ }^{64} \mathrm{Zn}$ system, $\mathrm{S}_{\alpha}$ is the $\alpha$ separation energy of the projectile and $\mathrm{m}_{\text {clu }}$ and $\mathrm{m}_{\text {proj }}$ are the masses of the captured cluster and of the projectile respectively. Excitation energies estimated for DCT were only slightly smaller than the ones for ICF, giving essentially the same relative yield as for ICF. A comparison between the calculations and the experimental ER relative yield, at two different energies, is shown in figure 3 for the collision ${ }^{6} \mathrm{Li}+{ }^{64} \mathrm{Zn}$. As one can see, above the Coulomb barrier $\left(\mathrm{V}_{\mathrm{B}} \sim 13 \mathrm{MeV}\right)$, the experimental relative yields are similar to the ones predicted by $\mathrm{CF}$ Cascade calculations, showing that $\mathrm{CF}$ is the dominating mechanism. However, at energies below the barrier, the HR experimental relative yield is rather different than the one expected for $\mathrm{CF}$, showing that $\mathrm{CF}$ is no longer the dominating reaction mechanisms while other processes such as ICF, DCT or single nucleon transfer become very important. As one can see in figure 3, 
for ${ }^{6} \mathrm{Li}+{ }^{64} \mathrm{Zn}$ the relative yield below the barrier is dominated by ${ }^{65} \mathrm{Zn}$ and ${ }^{65} \mathrm{Ga}$ which are not expected in CF but can be produced in ICF/DCT of a deuteron as well as in $1 \mathrm{n}$ and $1 \mathrm{p}$ transfer reactions respectively. Similar results were also observed for ${ }^{7} \mathrm{Li}+{ }^{64} \mathrm{Zn}$. Present results, suggesting the dominance of complete fusion above barrier in collision with weakly bound nuclei on medium-light targets, interestingly agree with our old fusion studies for the system ${ }^{13} \mathrm{~N}+{ }^{9} \mathrm{Be}$ concerning the collision of a weakly bound radioactive nucleus $\left(\mathrm{S}_{\mathrm{p}}\left({ }^{13} \mathrm{~N}\right) \approx 1.9 \mathrm{MeV}\right)$ onto a stable weakly bound $\left.\operatorname{target}\left(\mathrm{S}_{\mathrm{n}}{ }^{9} \mathrm{Be}\right) \approx 1.7 \mathrm{MeV}\right)$ [27]. Here the ER have been directly detected and identified using an array of Si telescopes, each integrated on a single silicon chip $[28,29]$ and, once more, their relative yield above barrier is very well reproduced by statistical model calculations for CF.

The discussed results results suggest that, in order to study fusion below the barrier in collisions induced by weakly bound nuclei on medium mass targets, the simple integration of the HR yield is not giving us the TF cross sections. Indeed different processes such as DCT and single neutron transfer can give an important contribution in the region below the barrier. Therefore, one needs to find experimental techniques allowing to properly separate the different reaction mechanisms contributing to the HR yield.
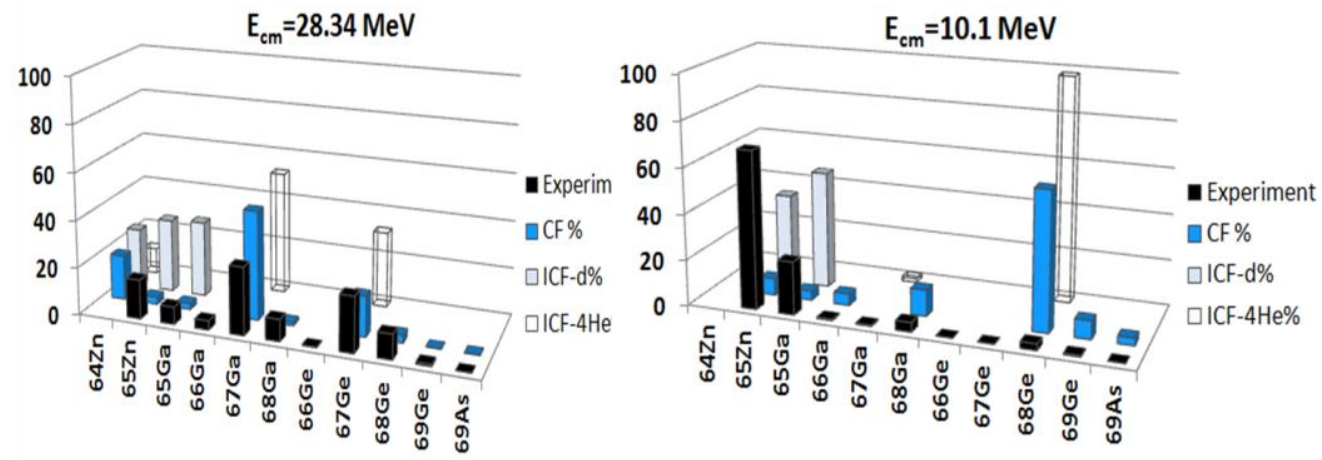

Figure 3. Experimental heavy residue relative yield for ${ }^{6} \mathrm{Li}+{ }^{64} \mathrm{Zn}$ at two different energies, compared with the prediction of statistical model calculations for complete fusion and deuteron or $\alpha$ capture.

\section{Summary and conclusions}

Fusion excitation functions in collisions induced by n-halo nuclei measured by different authors have been reported in the literature. Presently, available results suggest the presence of a fusion cross section enhancement around the barrier for n-halo nuclei when compared both to SBP calculations based on potentials which do not take into account the diffuse structure the projectile, or to the excitation functions induced by the halo nucleus core on the same targets. Most of the observed enhancement is removed if one compares reduced fusion excitation functions in order to eliminate static effects. A systematic study of many existing experimental data suggests that, for collisions induced by neutron halo nuclei, once static effects are removed, the residual effect of coupling to continuum or transfer is a suppression of fusion above barrier and, most probably, a residual small enhancement of fusion below the barrier.

Fusion has also been extensively studied in collisions induced by the stable weakly bound nuclei ${ }^{6} \mathrm{Li},{ }^{7} \mathrm{Li}$ and ${ }^{9} \mathrm{Be}$, having low breakup thresholds and a marked cluster structure of the ground state. In collision on heavy targets, were CF and ICF can be easily separated, data show a well established suppression of CF due to ICF following projectile breakup. In collisions with medium mass or light targets CF and ICF cannot be easily separated and most of available data refers to TF. Our ${ }^{6,7} \mathrm{Li}+{ }^{6} \mathrm{Zn}$ fusion data suggest that above barrier HR production is dominated 
by CF. Below the barrier, on the contrary, other processes such as ICF but also direct cluster transfer or single nucleon transfer are the dominating processes for HR production.

Although different aspects of the discussed topic have been clarified by the papers published so far, new better quality data are necessary for a more complete understanding to our opinion. Almost all the results obtained so far for collisions induced by halo nuclei have been obtained with ${ }^{6} \mathrm{He}$ beams and additional data with different halo nuclei would be necessary to build up a compete systematics. At the same time, new fusion cross sections measurements with halo nuclei, better exploring the sub-barrier region are still needed, since most of the data available do not explore this energy range with reasonable errors. New fusion data with proton halo nuclei, such as ${ }^{8} \mathrm{~B}$, are also necessary since no clear conclusion is reached in the literature on the effect of the p-halo on fusion.

In the study of collisions induced by weakly bound nuclei with no halo structure, new fusion cross sections measurements would be needed, especially on medium mass or light targets, at energies extending well below the barrier. These measurements should possibly be performed with experimental techniques allowing to clearly disentangle among the transfer, $\mathrm{CF}$ and ICF mechanisms leading to the production of the detected heavy residues.

As final remark, we wish to underline that measuring fusion excitation functions with activation techniques using a multiple target stack, allows to spare a large amount of beam time, but reliable accurate results can be extracted only taking properly into account both statistical energy straggling and target non uniformity effects.

\section{Acknowledgements}

The experimental results of our group, mentioned in the present review paper, have been obtained in different experiments involving many colleagues belonging to different institutions whose contribution is gratefully acknowledged.

\section{References}

[1] L. F. Canto et al., Phys. Rep. 424, (2006), 1

[2] N. Keeley et al., Prog. Part. Nucl. Phys. 59, (2007), 579

[3] N. Keeley et al., Prog. Part. Nucl. Phys. 63, (2009), 396

[4] A. Di Pietro et al., Europhys. Lett. 64, (2003), 309

[5] A. Di Pietro et al., Phys. Rev. Lett. 105, (2010), 022701

[6] M. Cubero et al., Phys. Rev. Lett. 109, (2012), 262701

[7] R.Raabe et al., Nature, 431, (2004), 823

[8] M. Alcorta et al., Phys. Rev. C 106, (2011), 172701

[9] V. Scuderi et al., Phys. Rev. C. 84, (2011), 064604

[10] Yu. E. Penionzhkevich et al., Eur. Phys. Jour. A 31, (2007), 185

[11] A. Lemasson et al., Phys. Rev. Lett. 103, (2009), 232701

[12] R. Wolski et al., Eur. Phys. Jour. A, 47, (2011), 111 
[13] P. A. DeYoung et al., Phys. Rev. C 58, (1998), 3442

[14] A. Di Pietro et al., Phys. Rev. C 87, (2013), 064614

[15] L. F. Canto et al., Nucl. Phys. A 821, (2009), 51

[16] P. R. S. Gomes et al., Phys. Rev. C 79, (2009), 027606

[17] E. F. Aguilera et al., Phys. Rev. Lett. 107, (2011), 092701

[18] A. Pakou et al., Phys. Rev. C 87, (2013), 014619

[19] J. Rangel et al., Eur. Phys. Jour. A 49, (2013), 57

[20] M. Dasgupta et al., Phys. Rev. C 70, (2004), 024606

[21] P.R.S. Gomes et al., Phys. Rev. C 73, (2006), 064606

[22] M. K. Pradhan et al., Phys. Rev. C 83, (2011), 064606

[23] M. Sinha et al., Eur. Phys. Jour. A 44, (2010), 403

[24] C. Beck et al., Phys. Rev. C 67, (2003), 054602

[25] M. Ray et al., Phys. Rev. C 78, (2008), 064617

[26] A. Di Pietro et al., Nucl. Phys. A 689, (2001), 668

[27] A.Di Pietro et al., Eur. Phys. Jour. ST 150, (2007), 15

[28] G. Cardella et al., Nucl. Instr. and Meth. A 378, (1996), 262

[29] F.Amorini et al., Nucl. Instr. and Meth. A 550, (2005), 248 